\title{
Uncertainty Model for Total Solar Irradiance Estimation on Australian Rooftops
}

\author{
Hassan Al-Saadi, Rastko Zivanovic \& Said Al-Sarawi \\ School of Electrical and Electronic Engineering, The University of Adelaide \\ Adelaide SA 5000, Australia. \\ hassan.al-saadi, rastko.zivanovic, said.alsarawi\}@adelaide.edu.au
}

\begin{abstract}
.
The installations of solar panels on Australian rooftops have been in rise for the last few years, especially in the urban areas. This motivates academic researchers, distribution network operators and engineers to accurately address the level of uncertainty resulting from gridconnected solar panels. The main source of uncertainty is the intermittent nature of radiation, therefore, this paper presents a new model to estimate the total radiation incident on a tilted solar panel. Where a probability distribution factorizes clearness index, the model is driven upon clearness index with special attention being paid for Australia with the utilization of bestfit-correlation for diffuse fraction. The assessment of the model validity is achieved with the adoption of four goodness-of-fit techniques. In addition, the Quasi Monte Carlo and sparse grid methods are used as sampling and uncertainty computation tools, respectively. High resolution data resolution of solar irradiations for Adelaide city were used for this assessment, with an outcome indicating a satisfactory agreement between actual data variation and model.
\end{abstract}

Keywords- Solar irradiation prediction, tilted surface, goodness of fit, diffuse fraction correlation.

\section{Introduction}

The current prevalence of solar rooftops in Australia incentivizes academic researchers, engineers and power network operators to investigate in detail solar engineering applications. As part of this tendency, solar irradiance prediction has been given a tremendous interest and revealed different approaches and methods. The importance of such studies lies in the fact that, to date, a small portion of Photovoltaic (PV) rooftops are equipped with real-time communication, measurement and control devices, resulting in increased cost [1]. Nonetheless, the majority of PV installations are not observable and controllable. While the availability of PV measurement and control devices is subject to high costs, it is difficult to collect the required specification information from each unit. Meanwhile, solar energy reaching the ground can be predicted with high accuracy 
by the utilization of statistical estimation functions at a given time for a predefined location, as discussed hereinafter. Therefore, obtaining a prior accurate knowledge on solar radiation can help in improving the quality of feed-in energy, reducing the ancillary costs of weather forecast equipment, mitigating the negative impacts of high PV penetration, especially in residential areas, storage quantifying and a better integration of PVs into the so-called "smart grid".

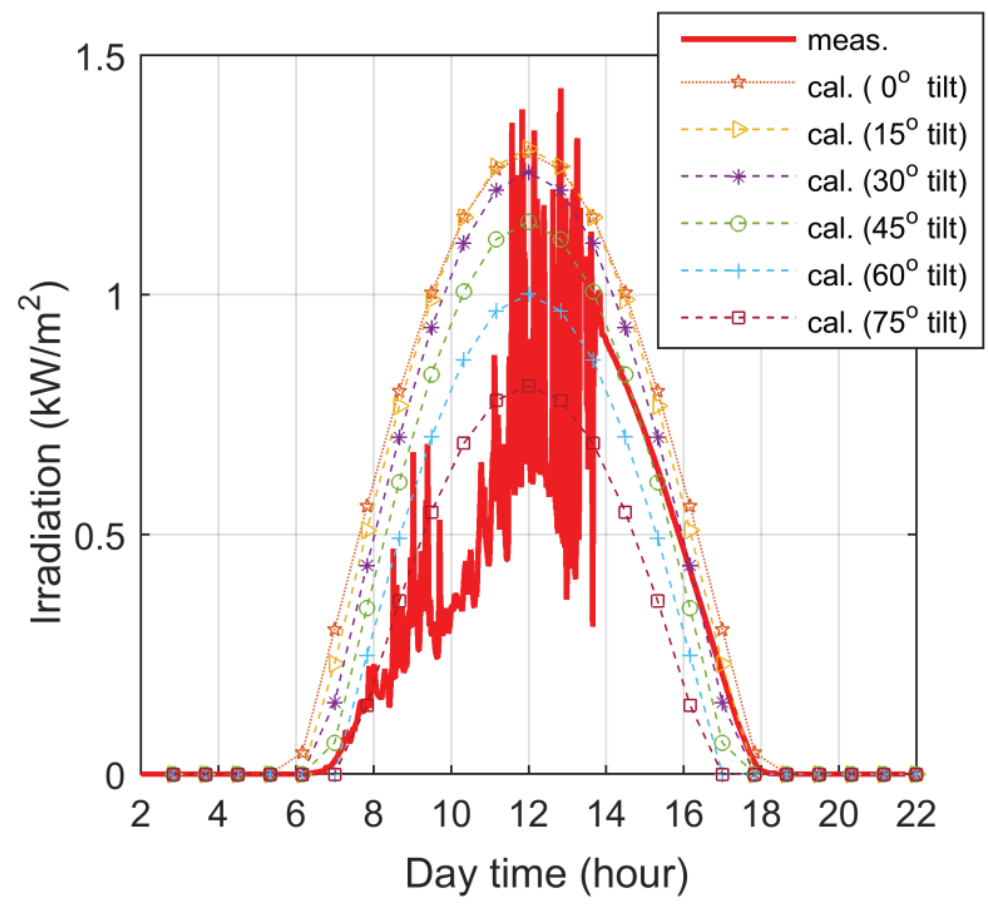

Figure 1 Measured data of one day in January 2016, compared with calculations of expected values of probabilistic irradiation model in hourly evaluations. 
The major step to overcome the adversity of uncertainty level is the use of several irradiance representation models in which strengths and weaknesses of each model can be examined as well as their complexity. In this paper, a new model for uncertainty prediction of solar irradiation incident on a tilted surface is presented. Where such model is yet to be satisfactorily modelled, this model is built upon a given characterization of clearness index and diffuse fraction of radiation while taking into consideration the Australian climate conditions. Since the index and the fraction are proven to have a functional relationship [2], the properties of clearness index can dictate the level of uncertainty in the total incident radiation. In this regard, the probabilistic evaluation of uncertainty, given probability density function (PDF) similar to the one given in [3], can specify the amount of the ground-reaching radiation.

The contribution of this work is to provide a simple and easy to use model that can be incorporated or adapted into other models to stimulate interdisciplinary research and system engineering applications. The uncertainty modelling of irradiation is presented in the following section with the emphasis on total irradiation estimation with the embedment of solar variation. In Section 3, four different statistical tools, namely root mean square error (RMSE), mean bias error (MBE), KolmogorovSmirnov test (KST) and correlation coefficient $\left(R^{2}\right)$ are used as part of this model assessment. Then, the implementation of the presented model is carried out and assessed with high resolution solar radiation data for two different seasonal days at several day times in Adelaide are discussed in Section 4. The last section presents a summary of this research outcomes.

\section{Irradiation Uncertainty Modelling \\ Total Irradiation}

The total radiation incident on a tilted surface, $I_{T}$, with the assumption of anisotropic sky, can be rewritten as:

$$
I_{T}=\frac{k_{t}}{I_{g}} \cdot\left[\left(I_{b} I_{o}+I_{d} I_{b}\right) R_{b}+I_{d}\left(I_{o}-I_{b}\right)\left(\frac{1+\cos \beta}{2}\right)\left[1+\sqrt{\frac{I_{b}}{I_{g}}} \sin ^{3} \frac{\beta}{2}\right]\right]+I_{g} \rho\left(\frac{1-\cos \beta}{2}\right) .
$$

In this model, circumsolar diffuse, horizon brightening as well as anisotropy factor are incorporated into the isotropic model resulting in the so called "HDKR" model, named after the initials of Hay, Davies, Klucher and Reindl researchers [4]. The $I_{o}$, $I_{g}, I_{b}$ and $I_{d}$ are the exterritorial, global, beam and diffuse irradiances, respectively; $R_{b}$ is Ratio of beam radiation on tilted surface to that on horizontal surface. The tilt 
angle is denoted by $\beta$ and the reflectance ground factor is $\rho . k_{t}$ is the hourly clearness index.

\section{Solar Variation}

For the variations of solar radiation, two vital factors have been identified to be representing the clearness of the sky: clearness index, $k_{t}$, and diffuse fraction, $k_{d}$. These quantities are highly correlated with different functions established on the subject, such as piecewise linear [2], piecewise $4^{\text {th }}$-order polynomial [5], piecewise $3^{\text {rd }}$-order polynomial [6], piecewise $2^{\text {nd }}$-order polynomial [7], logistic [8]. The logistic function is proven to be the best-fit based on Australian meteorological conditions. Later, the logistic function is further complicated adding more sensitive factors, for example, the effects of apparent solar time, persistence of global radiation level and daily clearness index. Providing the value of $k_{t}$, the $k_{d}$ can be computed using the logistic function as:

$$
k_{d}=\frac{1}{1+e^{-B\left(B \prime-k_{t}\right)}},
$$

where $B$ and $B^{\prime}$ are specified parameters for the logistic based diffuse fraction that can be computed as demonstrated in [8].

The behavior of $k_{t}$ is assumed to follow a special distribution with various approaches are proposed given the characteristics of the place, such as time standard meridian, number of the days in a year, longitude, latitude, altitude, and ground reflectance, further details can be found in [9]. In this paper, we choose to utilize the single Gamma distribution proposed by Holland and Huget [3] where the probability density is characterized by time and locations.

\section{Uncertainty Model}

As the discussed hereinbefore, it is possible to form an uncertainty model when the uncertainty behavior of $k_{t}$ is known. Therefore, after rewritten (1) to be a function of $k_{t}$ and $k_{d}$, the hourly total irradiation on a surface with tilt angle, $\beta$, can be expressed as:

$$
\begin{aligned}
I_{T}= & {\left[R_{b}(n, t)+k_{d}\left[-R_{b}(n, t)+k_{t} R_{b}(n, t)-k_{d} k_{t} R_{b}(n, t)+c\left(k_{d}\right)-k_{t} c\left(k_{d}\right)+\right.\right.} \\
& \left.\left.k_{d} k_{t} c\left(k_{d}\right)\right]+\rho\left(\frac{1-\cos \beta}{2}\right)\right] \times H_{o}(n) \times r_{d}(n, t),
\end{aligned}
$$

where 


$$
c\left(k_{d}\right)=\left(\frac{1+\cos \beta}{2}\right)\left[1+\sqrt{1-k_{d}} \sin ^{3} \frac{\beta}{2}\right] .
$$

The predefined quantities that function of $n$, which is the number of days, and $t$, day time, are shown with two brackets next to them while the uncertain quantity, $k_{t}$, is just indexed by $t$. In addition, the values of $H_{o}$ are just indexed by $n$ (cf. [4] \& [3] for extra information).

For the initial assessment of the model's results, Figure 1 shows the hourlycalculated total irradiations $\left(\mathrm{kW} / \mathrm{m}^{2}\right)$ for different tilt angles in a day for the city of Adelaide in comparison with the measured (one-minute) solar exposure data taken from the data portal webpage [10]. Herein, we utilize the Sparse Grid Technique [11] for the computations of the hourly expectation of $I_{T} .0$ to 1 is the chosen domain for $k_{t}$ as it is conventionally assumed. This is as the $k_{t}$ is the ratio of terrestrial to extraterrestrial radiation idealy this should have a maximum value of 1 , however if more influential factors are included such as the edges of the clouds the ratio could reach to 1.4 [9]. Regarding the figure and although the objectivity of assessing the model graphically is doubtable, a preliminary indication of the effectiveness of the present model can be drawn from these expectations.

\section{Model's Assessment}

The quality assessment of the modeled data is performed using goodness-of-fit techniques such as Root Mean Square Error (RMSE), Mean Bias Error (MBE), Coefficient of determination $R^{2}$ and Kolmogorov-Smirnov (KS) as in the following subsections. The usefulness of these metrics is to test the linear relationship between modelled and measured data.

\section{Root mean square error}

RMSE delivers a positive value with a tendency towards zero for ideal models. In the assessment of uncertainty models, the normalized version of RMSE with respect to predicted values is, herein, employed, as defined in [12] with objective justifications explained. Then, the short-term performance is evaluated as a smaller value means a better performance. The RMES value can be obtained using:

$$
\mathrm{RMSE}=\sqrt{\frac{1}{\mathrm{n}} \sum_{i=1}^{n} \frac{Y_{i, \text { calc. }}-Y_{i, \text { meas. }}}{Y_{i, \text { calc. }}} .}
$$

where $Y$ represents a set of data with the subscripts "calc." and "meas." represent calculated values from the model and measured values taken from meteorological stations. The subscript $i$ denotes the $i$-th value of an hourly solar irradiation; $n$ is the number of the hours from different days in a month across involved years. 


\section{Mean bias error}

The mean bias error (MBE) gives an indication of the average bias of the model. The value of MBE could be negative indicating the underestimation of tested model, as the desirable values are the low positive ones. Despite the popularity of this test, it is intuitively obvious that an overestimation may cancel an underestimation for individual observations, which is a well-recognized limitation of the MBS. According to [13], the formula of MBE with the normalization in respect to the calculated data can be expressed as:

$$
\mathrm{MBE}=\frac{1}{n} \sum_{i=1}^{n} \frac{Y_{i, \text { calc }}-Y_{i \text {,meas. }}}{Y_{i, \text { calc. }}},
$$

The descriptions of the subscripts $i, n$, calc. and meas. are mentioned in the previous subsection.

\section{Kolmogorov-Smirnov test}

The Kolmogorov-Smirnov test (KST) involves the use of cumulative density functions $(\mathrm{CDF}), F$, of both model and empirical data where the large difference is computed as follows:

$$
\mathrm{KST}=\sup _{1 \leq i \leq n}\left|F_{i, \text { meas. }}-F_{i, \text { calc. }}\right| \text {, }
$$

In (6), $F_{i, \text { meas. }}$ represents the CDF of empirical probability during $i$-th observation while $F_{i, c a l c}$. stands for the CDF of thereortical model probability at $i$-th sample. The smallest value of KST indicates the goodness of fit of the proposed model.

\section{The correlation coefficient $\left(R^{2}\right)$}

The correlation coefficient, $R^{2}$, is the determination of linear relations between calculated and measured observations. In this test, the variance of errors to the variance of the calculated data is compared, examining the quality of the model. It can be computed as follows:

$$
R^{2}=1-\frac{\sum_{i=1}^{n}\left(Y_{i, \text { meas }}-Y_{i, \text { calc. }}\right)^{2}}{\sum_{i=1}^{n}\left(Y_{\text {i,meas. }}-\bar{Y}_{\text {meas }}\right)^{2}},
$$


The descriptions of variable $Y$ and the subscripts $i, n$, calc. and meas. are mentioned in the previous subsection; and $\bar{Y}_{i, \text { meas. }}=\sum_{i=1}^{n} Y_{i \text {,meas. }}$. The desirable result of this test is that whenever its value is close to a positive integer one.

\section{Implementation and Discussion}

As the proposed uncertainty model involves hourly clearness index calculation, the computation of monthly average daily clearness index is necessary providing only monthly average solar irradiation. Therefore, two monthly quantities are used for

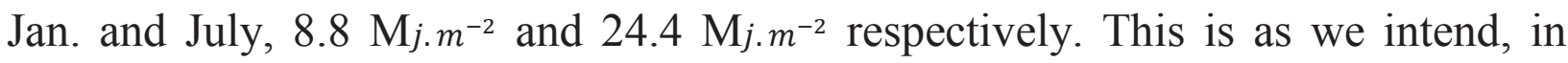
addition, to test the seasonal variations in the performance of the model. $\beta$ is chosen to be $30^{\circ}$ and $k_{t \min }$ and $k_{t \max }$ to be 0 and $1.4 \mathrm{pu}$, respectively. Moreover, ground reflectance is $0.2 \mathrm{pu}$. It is of importance to mention that for data sampling from the proposed model the Quasi Monte Carlo Method is utilized with the number of samplings is equal to the empirical data involved. In the meanwhile, the 1-min dataset of solar radiation can be obtained from Australian Bureau of Meteorology with the support of Australian Solar Institute (works under Australian Renewable Energy Agency - ARENA). The years considered in the current study are 95, 96, 97, $04,05,06,07,08,10,11,14,15,16$ for assessment of the model in January and 95, $96,97,04,05,06,07,08,10,11,14$, for the month July, resulting, for each hour, 24180 and 20460 measurements, respectively. The work is implemented using Matlab and MS Excel and run on Intel® i7-2600 4-core @ 3.4GHz processor.

In the implementation and assessment of the model, average hourly solar radiation is achieved for each consecutive minutes, which is the most common convention for characterizing solar radiations in short-time series analysis. From the presented simulation and result, it is clear that the presented model covers the most possible outcomes in solar radiation modelling in Australia. The statistics employing goodness-of-fit techniques mentioned in the previous section are shown in the

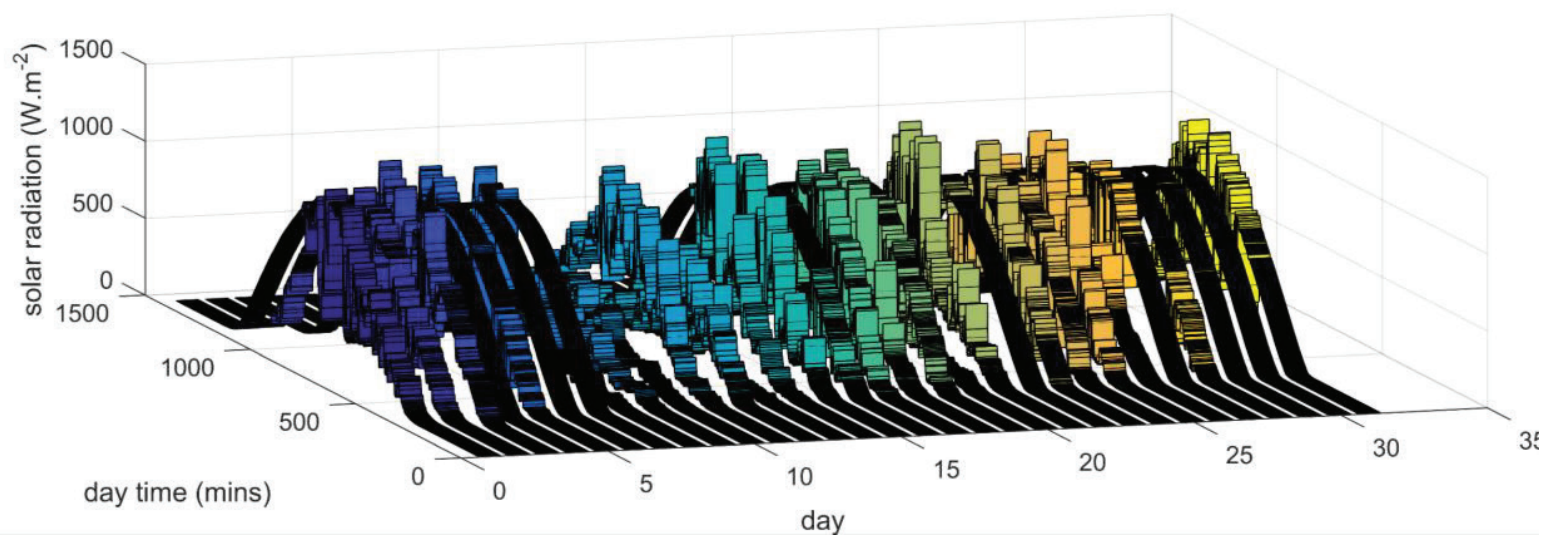

Figure 2 Empirical data of beam and diffuse solar radiations combined in Adelaide. 
Tables 1 and 2. In these two tables, model assessment accounted for seasonal variations are present where summer in Table 1 and winter in Table 2.

The statistical data from the assessment show various results as some are significantly noteworthy. The bolded results are distinguished among the rest to be desirable. It is clear that the presented modeling results are not consistent for individual day times, as in Table $1 \& 2$ (a), (b) or for $n$, as in Table $1 \& 2$ (c) has been given. However, there is a tendency that the model performs batter overall when $n=30$, according to RMSE. In terms of MBE, the statistics obtained for certain days distributed around the month, $(10,15,20,25,30)$, show an acceptable fit between measured data and modelled ones.

The outcome of the presented results are consistent with other studies such as in [14] where $\mp 10 \%$ is considered satisfactory. Based on the sign of MBE tests, no overestimation is noticed for the assessment of January while in July over-/underestimations are obvious. KST and $R^{2}$ are implemented and compared with RMSE and MBE as, in Table 1(a), KST shows similar assessment with RMSE and MBE. This is while in Table 2(b) the KST and $R^{2}$ are in agreement with MBE at 12:00PM. 
Table 1. Statistics obtained employing RMSE, MBE, $\mathbf{R}^{2}$ and KST for: a) different times of a day when $n=15$ of January, b) different times of a day when $n=30$ of January, c) an hour around noon of different $n$

(a) in January.

\begin{tabular}{l|lllr} 
Day time & RMSE & MBE & KST & $\boldsymbol{R}^{2}$ \\
\hline 09:00AM & 6.1535 & -0.9744 & 0.0164 & -1.9793 \\
10:00AM & 3.7191 & -0.6683 & $\mathbf{0 . 0 1 0 5}$ & -1.1501 \\
11:00AM & 4.7896 & -0.7512 & 0.0139 & -1.0727 \\
12:00PM & 4.5223 & -0.7629 & 0.0160 & -0.9765
\end{tabular}

(b)

\begin{tabular}{l|llll} 
Day time & RMSE & MBE & KST & $\boldsymbol{R}^{2}$ \\
\hline 09:00AM & 1.7170 & $-\mathbf{0 . 1 1 2 1}$ & 0.0174 & $-\mathbf{0 . 9 2 8 6}$ \\
$\mathbf{1 0 : 0 0 A M}$ & $\mathbf{1 . 0 8 2 2}$ & -0.1416 & 0.0127 & -1.1836 \\
$\mathbf{1 1 : 0 0 A M}$ & 1.4021 & -0.2211 & $\mathbf{0 . 0 1 2 1}$ & -1.0813 \\
$\mathbf{1 2 : 0 0 P M}$ & 1.3748 & -0.3022 & 0.0151 & -1.2591
\end{tabular}

(c)

\begin{tabular}{c|cccc}
$\boldsymbol{n}$ & RMSE & MBE & KST & $\boldsymbol{R}^{2}$ \\
\hline $\mathbf{5}$ & 33.3050 & -7.0233 & 0.0208 & -5.9079 \\
$\mathbf{1 0}$ & 8.5701 & -1.6641 & 0.0175 & -2.0678 \\
$\mathbf{1 5}$ & 3.9784 & -0.6463 & 0.0157 & -0.8369 \\
$\mathbf{2 0}$ & 2.3555 & -0.3349 & $\mathbf{0 . 0 1 4 9}$ & -0.5994 \\
$\mathbf{2 5}$ & 1.6203 & $-\mathbf{0 . 2 6 1 6}$ & 0.0150 & $-\mathbf{0 . 8 4 5 7}$ \\
$\mathbf{3 0}$ & $\mathbf{1 . 3 7 4 8}$ & -0.3022 & 0.0151 & -1.2591
\end{tabular}


Table 2. Statistics obtained employing RMSE, MBE, $\mathbf{R}^{2}$ and KST for: a) different times of a day when $n=15$ of July, b) different times of a day when $n=30$ of July., c) an hour around noon of different $n$ in July.

(a)

\begin{tabular}{l|llll}
\multicolumn{1}{c}{ Day time } & RMSE & MBE & KST & $\boldsymbol{R}^{2}$ \\
\hline 09:00AM & 5.4950 & $-\mathbf{0 . 5 6 6 8}$ & 0.0470 & -24.9514 \\
10:00AM & 8.4597 & -0.8117 & 0.0442 & -13.3852 \\
11:00AM & 4.9798 & -0.6498 & 0.0375 & -8.9466 \\
12:00PM & 8.1474 & -0.8518 & $\mathbf{0 . 0 3 5 2}$ & $\mathbf{- 7 . 2 3 4 1}$
\end{tabular}

(b)

\begin{tabular}{l|lllc} 
Day time & \multicolumn{1}{l}{ RMSE } & MBE & KST & $\boldsymbol{R}^{2}$ \\
\hline 09:00AM & 0.8828 & 0.6268 & 0.0452 & -28.1024 \\
10:00AM & 1.1153 & 0.5053 & 0.0432 & -14.8841 \\
11:00AM & $\mathbf{0 . 7 7 1 2}$ & 0.4496 & 0.0347 & -10.3655 \\
12:00PM & 1.0730 & $\mathbf{0 . 3 7 2 7}$ & $\mathbf{0 . 0 3 3 3}$ & $-\mathbf{- 9 . 3 5 4 5}$
\end{tabular}

(c)

\begin{tabular}{c|llll}
$\boldsymbol{n}$ & RMSE & MBE & KST & $\boldsymbol{R}^{2}$ \\
\hline $\mathbf{5}$ & 294.0569 & -45.9685 & 0.0394 & -19.5802 \\
$\mathbf{1 0}$ & 41.0301 & -6.4490 & 0.0362 & -11.8846 \\
$\mathbf{1 5}$ & 10.2086 & -1.2191 & 0.0353 & -7.7100 \\
$\mathbf{2 0}$ & 3.7727 & $-\mathbf{0 . 0 5 9 2}$ & 0.0348 & $-\mathbf{6 . 3 1 1 1}$ \\
$\mathbf{2 5}$ & 1.8125 & 0.2873 & 0.0341 & -6.9740 \\
$\mathbf{3 0}$ & $\mathbf{1 . 0 7 3 0}$ & 0.3727 & $\mathbf{0 . 0 3 3 3}$ & -9.3545
\end{tabular}

Overall, the objectiveness of the goodness-of-fit techniques proves the acceptable quality of the proposed model. However, due to the limited range of empirical data to within few years a few "non-desirable" values of RMSE, MBE, $R^{2}$ and KST can be seen. Indicating that for a more accurate modelling there is a need to have fully evaluate solar prediction model for more years. 


\section{Conclusion}

This paper presents a new uncertainty model for the estimation of total solar radiation reaching Australian rooftops. The uncertainty is characterized by the PDF of clearness index when the diffuse fraction is functionally correlated through the utilization of logistic relation. Four assessment techniques are employed to examine the effectiveness of the model with the involvement of high resolution data recorded for Adelaide.

The assessment covers the day hours for two different days taken seasonal variations into consideration. In this assessment, different day times give different outcomes when an hour around noon and three before noon. However, there is an agreement with root mean square error (RMSE), mean bias error (MBE) and KolmogorovSmirnov test (KST) in evaluating the model during the hour around 10:00AM when the number of the day is 15 . While the agreement is apparent for MBE, KST and the correlation coefficient $\left(R^{2}\right)$ when $12: 00 \mathrm{PM}$ at 30 as a day's number in the model. Although the agreements do not exactly occur in all times and days, other assessment results are still within the acceptable range comparing with other work, mentioned in the main scripts.

The considerations that should be paid for future work are more assessments on other Australian cities. Moreover, Expanding the time domain with different day times could yield more reliable outcomes of the proposed model. 


\section{References}

[1] L. Cibulka, M. Brown, L. Miller and A. V. Meier, "User requirements and research needs for renewable generation forecasting tools that will meet the needs of the caiso and utilities for 2020," in A White Paper Report Prepared by CIEE, 2012.

[2] J. F. Orgill and K. G. T. Hollands, "Correlation equation for hourly diffuse radiation on a horizontal surface," Sol. Energy, vol. 19, no. 4, pp. 357-359, Dec. 1977.

[3] K. G. T. Hollands and R. G. Huget, "A probability density function for the clearness index, with applications," Solar Energy, vol. 30, no. 3, p. 195-209, Dec. 1983.

[4] J. A. Duffie and W. A. Beckman, Solar engineering of thermal processes, vol. 3, New York: Wiley, pp. ???, 2013.

[5] J. Chandrasekaran and S. Kumar, "Hourly diffuse fraction correlation at a tropical location," Sol. Energy, vol. 53, no. 6, pp. 505-510, Dec. 1994.

[6] S. Karatasou, M. Santamouri and V. Geros, "Analysis of experimental data on diffuse solar radiation in Athens, Greece, for building applications," Int. J. Sustain. Energy, vol. 23, no. (1-2), pp. 1-11, Mar. 2003.

[7] J. Soares, A. P. Oliveira, M. Z. Božnar, P. Mlakar, J. F. Escobedo and A. J. Machado, "Modeling hourly diffuse solar-radiation in the city of São Paulo using a neural-network technique," Appl. Energy, vol. 79, no. 2, pp. 201-214, Oct. 2004.

[8] J. Boland, B. Ridley and B. Brown, "Models of diffuse solar radiation," Renew. Energy, vol. 33, no. 4, pp. 575-584, Apr. 2008.

[9] K. T. Hollands and H. Suehrcke, "A three-state model for the probability distribution of instantaneous solar radiation, with applications." Solar Energy, vol. 96, pp. 103-112, Oct. 2013.

[10] [Online]. Available: http://reg.bom.gov.au/climate/reg/oneminsolar/index.shtml, last accessed $12^{\text {th }}$ of Dec, 2016.

[11] T. Gerstner and M. Griebel, "Numerical Integration Using Sparse Grids," Numerical Algorithms, vol. 18, no. 3-4, pp. 209-232, Jan. 1998.

[12] L. Martín, L. F. Zarzalejo, J. Polo, A. Navarro, R. Marchante and M. Cony, "Prediction of global solar irradiance based on time series analysis: application to solar thermal power plants energy production planning," Solar Energy, vol. 84, no. 10, pp. 1772-1781, 2010.

[13] T. E. Hoff, R. Perez, J. Kleissl, D. Renne and J. Stein, "Reporting of irradiance modeling relative prediction errors," Progress in Photovoltaics: Research and Applications, vol. 21, no. 7, pp. 1514-1519, 2013.

[14] Robaa, S. M. "Evaluation of sunshine duration from cloud data in Egypt," Energy 33.5 (2008): 785-795.

[15] B. Ridley, J. Boland and P. Lauret, "Modelling of diffuse solar fraction with multiple predictors," Renew. Energy, vol. 35, no. 2, pp. 478-483, Feb. 2010. 